\title{
Estado da arte: Práticas corporais de aventura e Educação Física escolar
}

\author{
State of the Art: Adventure body practices and Physical Education classes
}

\author{
Alexander Klein Tahara ${ }^{1, *}$ \\ Dandara de Carvalho Soares ${ }^{2}$ \\ Suraya Cristina Darido ${ }^{2}$
}

\section{Resumo}

Objetivo: Analisar a produção acadêmica sobre as práticas corporais de aventura (PCA) em periódicos científicos nacionais, visando qualificar e quantificar o número total de artigos publicados sobre esta temática, bem comoverificarosestudos que estãorelacionados à área da EducaçãoFísica escolar. Métodos: A pesquisa é de naturezaquali-quantitativa, sendofeitaumabuscana base de dados online de onzeperiódicoscientíficosnacionais, durante o recorte temporal de 2005 a 2017. Resultados: O estado da arteindica que a produção está mais incidente na relação das PCA com os aspectos biodinâmicos, seguido pela educação ambiental e variáveis psicológicas, e que ainda há uma carência na produção científica relacionada às PCA. Conclusão: Acredita-se que há necessidade de realização de mais estudos e pesquisas que incidam foco nas PCA como um conteúdo a ser ministrado nas aulas de Educação Física escolar.

Palavras-chave: estado da arte, práticas corporais de aventura, Educação Física escolar.

\begin{abstract}
Objective: To analyze the academic production on adventure body practices (PCA) in national scientific journals, aiming to qualify and quantify the total number of articles published on this subject, as well as to verify the studies that are related to the area of Physical Education school. Methods: The research is qualitative and quantitative, and a search was made in the online database of eleven national scientific journals, during the temporal cut from 2005 to 2017. Results: The state of the art indicates that production is more incident in relation of PCA with biodynamic aspects, followed by environmental education and psychological variables, and that there is still a lack in the scientific production related to PCA. Conclusion: It is believed that there is a need for further studies and research focusing on PCA as a content to be taught in the School Physical Education classes.
\end{abstract}

Keywords: state of the art, adventure body practices, Physical Education classes.
Afiliação dos autores

${ }^{1}$ Universidade Estadual de Santa Cruz, Ilhéus, Bahia, Brasil

Universidade Estadual Paulista, Rio Claro, São Paulo, Brasil

${ }^{*}$ Autor correspondente

Departamento de Ciências da Saúde DCSau, Campus Soane Nazaré de Andrade, Rodovia Jorge Amado, Km 16, Bairro Salobrinho, CEP: 45662-900, Ilhéus, Bahia, Brasil. e-mail: alexipatinga@yahoo.com

Conflito de interesses

Os autores declararam não haver conflito de interesses.

Processo de arbitragem

Recebido: 06/06/2018 Aprovado: 16/09/2018 


\section{Introdução}

Torna-se perceptível nos últimos anos o desenvolvimento de pesquisas que enfatizam o "estado da arte" na área da Educação Física escolar, entre eles Ontañón, Duprat e Bortoleto ${ }^{1}$ e as atividades circenses, Rufino e Darido $^{2}$ e a pesquisa-ação, Magrin, Simões e Moreira $^{3}$ e a formação profissional, Impolcetto e Darido e o conteúdo do voleibol, entre outros.

Tomando como referência as diretrizes propostas pela Base Nacional Comum Curricular ${ }^{5}$ (BNCC), em relação ao componente curricular "Educação Física", as Práticas Corporais de Aventura (PCA) constituem-se como uma das unidades temáticas a serem tratadas ao longo do Ensino Fundamental, entre os $6^{\circ}$ e $9^{\circ}$ anos (os $6^{\circ}$ e $7^{\circ}$ anos com as PCA urbanas, e os $8^{\circ}$ e $9^{\circ}$ anos com as PCA na natureza).

Ressalta-se que alguns trabalhos, como Pereira e Armbrust ${ }^{6}$, Franco, Cavasini e Darido, Maldonado e Silva ${ }^{8}$, Tahara, Cagliari e Darido ${ }^{9}$, entre outros, tecem reflexões acerca da relação que se estabelece entre as PCA enquanto um conteúdo a ser desenvolvido pelos professores em aulas de Educação Física escolar.

Assim sendo, o objetivo deste "estado da arte" foi analisar a produção acadêmica sobre as PCA em onze periódicos científicos nacionais, visando qualificar e quantificar o número total de artigos publicados sobre esta temática, bem como refletir a respeito dos estudos que estão relacionados à área da Educação Física escolar.

\section{Métodos}

Esta pesquisa é de natureza quali-quantitativa, assim como destaca Creswell ${ }^{10}$, no sentido de que nesta abordagem o pesquisador tende a basear seus pressupostos em campos pragmáticos, empregando estratégias que envolvem a coleta de dados tanto simultaneamente quanto sequencialmente, para melhor entender os problemas de pesquisa. A coleta e análise dos envolvem tanto informações numéricas quanto informações textuais, no sentido de proporcionar uma melhor compreensão do universo pesquisado.

A análise teve como recorte temporal os últimos treze anos, compreendido entre os anos de 2005 e 2017. Justifica-se tal recorte pelo fato de ter sido anos importantes para a área no sentido de haver disponível uma maior quantidade de artigos e trabalhos que mencionam de alguma forma as PCA, sendo que as discussões e reflexões acerca do tema se tornaram mais nítidas quando comparadas com anos anteriores ao do recorte temporal especificado.

Para tanto, inicialmente foi feita uma busca na base de dados online de onze periódicos nacionais, todos reconhecidos pelo portal Qualis/Capes - Plataforma Sucupira e classificados com estrato entre A2 e B4 (a data de referência de acesso ao portal pela última vez corresponde ao mês de dezembro de 2017), visando obter o número de artigos que remetem às PCA em cada uma destas revistas.

Ressalta-se que todos os periódicos são da área da Educação Física e mantém como diretrizes nos seus respectivos escopos a publicação de artigos nas mais diversas vertentes da Educação Física, do esporte e do movimento humano. As seguintes revistas foram analisadas: Revista Movimento (ESEF - UFRGS), estrato A2; Revista Motriz (DEF/UNESP - Rio Claro), estrato B1; Revista Brasileira de Ciências do Esporte (CBCE), estrato B1; Revista da Educação Física (UEM), estrato B1; Revista Brasileira de Educação Física e Esporte (RBEFE/USP), estrato B1; Motrivivência (UFSC), estrato B2; Revista Brasileira de Ciência e Movimento (RBCM), estrato B2; Pensar a Prática (FEFD - UFG), estrato B2; Conexões (FEFUNICAMP), estrato B4; Arquivos em Movimento (EEFD - UFRJ), estrato B4; Revista Mackenzie de Educação Física e Esporte (REMEFE), estrato B3.

A pesquisa na base de dados foi feita analisando-se todas as edições, tanto o título, o resumo, assim como, o corpo do texto em si, em cada um destes periódicos. Todos os artigos que apresentaram algum aspecto que remetesse às práticas de aventura foram considerados para eventual análise.

\section{Resultados e Discussão}

Os resultados encontrados estão apresentados natabela 1,0 qual ilustra o número de artigos totais publicados pelos onze periódicos (anualmente e no recorte temporal total), sendo no primeiro parênteses o número de artigos que retratam a temática da aventura de forma geral, e no segundo parênteses a quantidade que enfocam a relação da aventura com a área da Educação Física escolar.

Tabela 1

"Estado da Arte" e o conteúdo das PCA.

\begin{tabular}{|c|c|c|c|c|c|c|c|c|c|c|c|c|}
\hline $\begin{array}{l}\text { Periódico } \\
\text { /Ano }\end{array}$ & Movimento & Motriz & $\begin{array}{l}\text { REF } \\
\text { (UEM) }\end{array}$ & RBCE & $\begin{array}{c}\text { RBEFE } \\
\text { (USP) }\end{array}$ & $\mathrm{RBCM}$ & Motrivivêcia & $\begin{array}{c}\text { Pensar } \\
\text { a } \\
\text { Prática }\end{array}$ & Conexões & $\begin{array}{l}\text { Arquivos } \\
\text { em } \\
\text { Movimento }\end{array}$ & Mackenzie & $\begin{array}{c}\text { Total (por } \\
\text { ano) }\end{array}$ \\
\hline 2005 & $\begin{array}{c}30(1) \\
(0)\end{array}$ & $\begin{array}{c}25(0) \\
(0)\end{array}$ & $\begin{array}{c}24(1) \\
(0)\end{array}$ & $\begin{array}{c}33(0) \\
(0)\end{array}$ & $\begin{array}{c}28(0) \\
(0)\end{array}$ & $\begin{array}{c}57(2) \\
(0)\end{array}$ & $\begin{array}{c}22(0) \\
(0)\end{array}$ & $\begin{array}{c}17(0) \\
(0)\end{array}$ & $\begin{array}{c}15(0) \\
(0)\end{array}$ & $\begin{array}{c}20(0) \\
(0)\end{array}$ & $\begin{array}{c}10(0) \\
(0)\end{array}$ & $281(4)(0)$ \\
\hline 2006 & $\begin{array}{c}29(0) \\
(0)\end{array}$ & $\begin{array}{c}35(3) \\
(0)\end{array}$ & $\begin{array}{c}24(0) \\
(0)\end{array}$ & $\begin{array}{c}36(1) \\
(0)\end{array}$ & $\begin{array}{c}28(1) \\
(0)\end{array}$ & $\begin{array}{c}51(0) \\
(0)\end{array}$ & $\begin{array}{c}21(0) \\
(0)\end{array}$ & $\begin{array}{c}21(2) \\
(0)\end{array}$ & $\begin{array}{c}17(0) \\
(0)\end{array}$ & $\begin{array}{c}17(0) \\
(0)\end{array}$ & $\begin{array}{c}33(0) \\
(0)\end{array}$ & $312(7)(0)$ \\
\hline 2007 & $\begin{array}{c}31(0) \\
(0)\end{array}$ & $\begin{array}{c}38(0) \\
(0)\end{array}$ & $\begin{array}{c}24(0) \\
(0)\end{array}$ & $\begin{array}{c}40(7) \\
(1)\end{array}$ & $\begin{array}{c}35(0) \\
(0)\end{array}$ & $\begin{array}{c}57(0) \\
(0)\end{array}$ & $\begin{array}{c}22(0) \\
(0)\end{array}$ & $\begin{array}{c}22(0) \\
(0)\end{array}$ & $\begin{array}{c}18(0) \\
(0)\end{array}$ & $\begin{array}{c}20(1) \\
(0)\end{array}$ & $\begin{array}{c}55(1) \\
(0)\end{array}$ & $362(9)(1)$ \\
\hline 2008 & $\begin{array}{c}33(2) \\
(0)\end{array}$ & $\begin{array}{c}58(0) \\
(0)\end{array}$ & $\begin{array}{c}60(0) \\
(0)\end{array}$ & $\begin{array}{c}39(0) \\
(0)\end{array}$ & $\begin{array}{c}28(1) \\
(0)\end{array}$ & $\begin{array}{c}50(2) \\
(0)\end{array}$ & $\begin{array}{c}40(1) \\
(0)\end{array}$ & $\begin{array}{c}33(1) \\
(0)\end{array}$ & $\begin{array}{c}24(1) \\
(0)\end{array}$ & $\begin{array}{c}23(0) \\
(0)\end{array}$ & $\begin{array}{c}55(1) \\
(0)\end{array}$ & $443(9)(0)$ \\
\hline 2009 & $\begin{array}{c}54(3) \\
(0)\end{array}$ & $\begin{array}{c}110(3) \\
(0)\end{array}$ & $\begin{array}{c}60(0) \\
(0)\end{array}$ & $\begin{array}{c}42(1) \\
(0)\end{array}$ & $\begin{array}{c}34(2) \\
(0)\end{array}$ & $\begin{array}{c}55(3) \\
(0)\end{array}$ & $\begin{array}{c}23(0) \\
(0)\end{array}$ & $\begin{array}{c}39(0) \\
(0)\end{array}$ & $\begin{array}{c}28(0) \\
(0)\end{array}$ & $\begin{array}{c}20(1) \\
(0)\end{array}$ & $\begin{array}{c}33(0) \\
(0)\end{array}$ & $\begin{array}{c}498(13) \\
(0)\end{array}$ \\
\hline 2010 & $\begin{array}{c}66(1) \\
(0)\end{array}$ & $\begin{array}{c}119(7) \\
(0)\end{array}$ & $\begin{array}{c}60(0) \\
(0)\end{array}$ & $\begin{array}{c}62(3) \\
(0)\end{array}$ & $\begin{array}{c}48(1) \\
(0)\end{array}$ & $\begin{array}{c}57(4) \\
(0)\end{array}$ & $\begin{array}{c}36(0) \\
(0)\end{array}$ & $\begin{array}{c}42(1) \\
(0)\end{array}$ & $\begin{array}{c}42(0) \\
(0)\end{array}$ & $\begin{array}{c}20(0) \\
(0)\end{array}$ & $\begin{array}{c}32(0) \\
(0)\end{array}$ & $\begin{array}{c}584(17) \\
(0)\end{array}$ \\
\hline 2011 & $\begin{array}{c}60(0) \\
(0)\end{array}$ & $\begin{array}{c}84(2) \\
(0)\end{array}$ & $\begin{array}{c}60(1) \\
(0)\end{array}$ & $\begin{array}{c}70(3) \\
(0)\end{array}$ & $\begin{array}{c}72(3) \\
(0)\end{array}$ & $\begin{array}{c}52(0) \\
(0)\end{array}$ & $\begin{array}{c}33(0) \\
(0)\end{array}$ & $\begin{array}{c}46(3) \\
(0)\end{array}$ & $\begin{array}{c}24(1) \\
(0)\end{array}$ & $\begin{array}{c}16(1) \\
(1)\end{array}$ & $\begin{array}{c}24(0) \\
(0)\end{array}$ & $\begin{array}{c}541(14) \\
(1)\end{array}$ \\
\hline 2012 & $\begin{array}{c}60(1) \\
(1)\end{array}$ & $\begin{array}{c}77(0) \\
(0)\end{array}$ & $\begin{array}{c}60(0) \\
(0)\end{array}$ & $\begin{array}{c}66(1) \\
(0)\end{array}$ & $\begin{array}{c}63(0) \\
(0)\end{array}$ & $\begin{array}{c}56(1) \\
(0)\end{array}$ & $\begin{array}{c}39(0) \\
(0)\end{array}$ & $\begin{array}{c}66(0) \\
(0)\end{array}$ & $\begin{array}{c}54(0) \\
(0)\end{array}$ & $\begin{array}{c}16(2) \\
(0)\end{array}$ & $\begin{array}{c}25(1) \\
(0)\end{array}$ & $582(6)(1)$ \\
\hline 2013 & $\begin{array}{c}60(0) \\
(0)\end{array}$ & $\begin{array}{c}97(2) \\
(0)\end{array}$ & $\begin{array}{c}60(3) \\
(0)\end{array}$ & $\begin{array}{c}69(2) \\
(0)\end{array}$ & $\begin{array}{c}60(0) \\
(0)\end{array}$ & $\begin{array}{c}80(2) \\
(0)\end{array}$ & $\begin{array}{c}39(1) \\
(1)\end{array}$ & $\begin{array}{c}79(1) \\
(0)\end{array}$ & $\begin{array}{c}44(1) \\
(0)\end{array}$ & $\begin{array}{c}14(0) \\
(0)\end{array}$ & $\begin{array}{c}30(0) \\
(0)\end{array}$ & $\begin{array}{c}632(12) \\
(1)\end{array}$ \\
\hline 2014 & $\begin{array}{c}88(0) \\
(0)\end{array}$ & $\begin{array}{c}60(0) \\
(0)\end{array}$ & $\begin{array}{c}60(1) \\
(0)\end{array}$ & $\begin{array}{c}63(0) \\
(0)\end{array}$ & $\begin{array}{c}60(1) \\
(0)\end{array}$ & $\begin{array}{c}80(1) \\
(0)\end{array}$ & $\begin{array}{c}43(0) \\
(1)\end{array}$ & $\begin{array}{c}82(1) \\
(0)\end{array}$ & $\begin{array}{c}38(1) \\
(0)\end{array}$ & $\begin{array}{c}17(0) \\
(0)\end{array}$ & $\begin{array}{c}30(0) \\
(0)\end{array}$ & $621(5)(1)$ \\
\hline 2015 & $\begin{array}{c}75(2) \\
(0)\end{array}$ & $\begin{array}{c}54(0) \\
(0)\end{array}$ & $\begin{array}{c}60(2) \\
(0)\end{array}$ & $\begin{array}{c}57(1) \\
(0)\end{array}$ & $\begin{array}{c}60(1) \\
(0)\end{array}$ & $\begin{array}{c}80(1) \\
(0)\end{array}$ & $\begin{array}{c}52(0) \\
(0)\end{array}$ & $\begin{array}{c}78(1) \\
(0)\end{array}$ & $\begin{array}{c}48(0) \\
(0)\end{array}$ & $\begin{array}{c}12(0) \\
(0)\end{array}$ & $\begin{array}{c}15(1) \\
(0)\end{array}$ & $591(9)(0)$ \\
\hline 2016 & $\begin{array}{c}100(1) \\
(0)\end{array}$ & $\begin{array}{c}61(0) \\
(0)\end{array}$ & $\begin{array}{c}60(0) \\
(0)\end{array}$ & $\begin{array}{c}56(1) \\
(0)\end{array}$ & $\begin{array}{c}63(0) \\
(0)\end{array}$ & $\begin{array}{c}80(0) \\
(0)\end{array}$ & $\begin{array}{c}55(2) \\
(1)\end{array}$ & $\begin{array}{c}77(1) \\
(0)\end{array}$ & $\begin{array}{c}28(1) \\
\text { (2) }\end{array}$ & $\begin{array}{c}15(0) \\
(1)\end{array}$ & $\begin{array}{l}\text { Nada } \\
\text { consta }\end{array}$ & $595(6)(4)$ \\
\hline 2017 & $\begin{array}{c}75(3) \\
(0)\end{array}$ & $\begin{array}{c}47(0) \\
(0)\end{array}$ & $\begin{array}{c}60(1) \\
(0)\end{array}$ & $\begin{array}{c}44(1) \\
(0)\end{array}$ & $\begin{array}{c}16(0) \\
(0)\end{array}$ & $\begin{array}{c}60(0) \\
(0)\end{array}$ & $\begin{array}{c}50(0) \\
(1)\end{array}$ & $\begin{array}{c}53(0) \\
(0)\end{array}$ & $\begin{array}{c}17(0) \\
(0)\end{array}$ & $\begin{array}{l}\text { Nada } \\
\text { consta }\end{array}$ & $\begin{array}{l}\text { Nada } \\
\text { consta }\end{array}$ & $422(5)(1)$ \\
\hline $\begin{array}{l}\text { Total (por } \\
\text { periódico) }\end{array}$ & $\begin{array}{c}761(14) \\
(1)\end{array}$ & $\begin{array}{c}865 \\
(17)(0)\end{array}$ & $\begin{array}{c}672(9) \\
(0)\end{array}$ & $\begin{array}{c}677 \\
(21)(1)\end{array}$ & $\begin{array}{c}595(10) \\
(0)\end{array}$ & $\begin{array}{c}815 \\
(16)(0)\end{array}$ & $\begin{array}{c}475 \\
(4)(4)\end{array}$ & $\begin{array}{c}655 \\
(11)(0)\end{array}$ & $\begin{array}{c}397 \\
(5)(2)\end{array}$ & $\begin{array}{c}210 \\
(5)(2)\end{array}$ & $\begin{array}{c}342 \\
(4)(0)\end{array}$ & $\begin{array}{c}6464 \\
(116)(10)\end{array}$ \\
\hline
\end{tabular}

Do total de 6464 artigos encontrados na soma dos onze periódicos analisados durante o recorte temporal estipulado (anos de 2005 até 2017), 126 artigos (1,95\% do total) eram referentes às PCA. E destes 126 artigos, 10 trabalhos retratavam as PCA no universo das aulas de Educação Física escolar. 
Considera-se a grande representatividade das PCA na atualidade, especialmente no âmbito competitivo, onde são realizadas inúmeras competições em distintas modalidades, assim como também no âmbito turístico/lazer, em que as pessoas muitas vezes pagam por pacotes e viagens com o propósito de desfrutar da vivência de determinado tipo de prática de aventura.

Assim sendo, em relação à quantidade no número de artigos encontrados, acredita-se que a temática das PCA necessita de mais discussões e pesquisas científicas, ainda mais se pensando no âmbito educacional e no quanto tais práticas podem (e devem) estar inseridas ao contexto das aulas de Educação Física escolar.

Após a quantificação do número de artigos em cada um dos onze periódicos analisados e do número total de artigos encontrados no recorte temporal, houve a classificação destes trabalhos que remeteram aos enfoques dados às práticas de aventura.

Diante dos dados expressos natabela 2, é possível observar que ao realizar a categorização temática dos artigos referentes às PCA, abriram-se possibilidades de verificar quais são os temas dentro das PCA mais discutidos cientificamente em âmbito nacional.

Tabela 2

Categorização temática dos artigos que retratam as PCA.

\begin{tabular}{lc}
\hline \multicolumn{1}{c}{ Categorização dos Temas } & $\begin{array}{c}\text { Número de incidência nos } \\
\text { periódicos }\end{array}$ \\
\hline Biodinâmica & 27 \\
Educação Ambiental & 16 \\
Aspectos psicológicos & 13 \\
(estados emocionais) & 12 \\
Lazer & 12 \\
Risco & 10 \\
PCA na Educação Física escolar & 8 \\
Representações Sociais & 8 \\
Educação Física e Meio Ambiente & 6 \\
Formação profissional & 6 \\
Gênero & 6 \\
Histórico/Evolução & 4 \\
Metodologia de Ensino & 4 \\
Ética e Valores & 3 \\
Produção Acadêmica/Científica & 2 \\
Pessoas com necessidades especiais & 2 \\
(PNE) & 8 \\
Iniciação Esportiva & 147 \\
Outras categorias & \\
Total & \\
\hline
\end{tabular}

Ressalta-se que a abordagem e o enfoque de alguns artigos se encaixavam em duas ou mais categorias temáticas e, por isso, o total de 147 categorizações listadas na tabela foi superior ao total de artigos (126) que enfatizavam as práticas de aventura, seja de forma geral e em outros focos de estudo (116), ou relacionada à área da Educação Física escolar (10).

Houveram oito indicações para "Outras categorias", superior em relação a algumas categorias. Tal superioridade deve ao fato de que foram reunidas todas as categorias que tiveram apenas uma única aparição entre todos os artigos analisados. São elas: Cinema; Organizações e Federações; Questões Terminológicas; Demanda Potencial e Mercado; Idosos e a Aventura; Qualidade de Vida; Políticas Públicas e, por fim, Tratamento e dependência química.

Percebe-se que as temáticas mais expressivas e discutidas nos artigos estão relacionadas aos aspectos biodinâmicos atrelados às PCA seguido pelas questões referentes à educação ambiental. $\mathrm{Na}$ sequência, houve certo equilíbrio entre os aspectos psicológicos / estados emocionais, o lazer e o risco nas diferentes práticas.

Em relação aos aspectos biodinâmicos atrelados às PCA, pesquisas como Costa e Oliveira ${ }^{11}$, Tremea, Gomes e Loss ${ }^{12}$, entre outros, enfatizam de que forma tais práticas vêm sendo estudadas, por exemplo, em áreas como a Fisiologia, Biomecânica e Controle Motor.

Conforme salientam Manoel e Carvalho $^{13}$, a análise das áreas de concentração dos programas de pós-graduação permite identificar três subáreas: a biodinâmica, a sociocultural e a pedagógica. A biodinâmica se sobressai pela dimensão do corpo docente e pela quantidade de linhas e projetos de pesquisa, sempre mais numerosos em comparação com as outras duas subáreas. Tal hegemonia expressa a valorização atribuída às pesquisas orientadas pelas ciências naturais em detrimento daquelas fundamentadas pelas ciências humanas e sociais, além da dificuldade em problematizar a intervenção, particularmente no âmbito da escola.

No que tange às questões relacionadas à educação ambiental, estudos como Rosa e Carvalhinho ${ }^{14}$, Figueiredo e Schwartz ${ }^{15}$, entre outros, realçam a importância de se trabalhar a educação ambiental através da discussão e vivência das práticas corporais de aventura.

De maneira semelhante, em detrimento aos aspectos emocionais e variáveis psicológicas, estudos como Brandt et al. $^{16}$, Vieira et al. ${ }^{17}$, entre outros, despertam olhares a respeito das relações entre os aspectos psicológicos e os diferentes tipos de PCA.

Com resultado um pouco menos expressivo, está a categoria das PCA em aulas de Educação Física escolar. Atabela 3 evidencia quais foram os 10 trabalhos encontrados no "estado da arte" e que retratam (de maneira teórica e/ou prática) a relação entre as PCA e a Educação Física escolar.

\section{Tabela 3}

Trabalhos coletados no estado da arte que tratam da relação entre as PCA e a Educação Física escolar.

\begin{tabular}{|c|c|c|}
\hline Trabalho/Autores & Natureza & Descrição \\
\hline Guimarães et al. ${ }^{18}$ & Teórica & $\begin{array}{l}\text { EF no Ensino Médio e discussões sobre } \\
\text { Meio Ambiente / esportes de aventura }\end{array}$ \\
\hline Franco et al. ${ }^{19}$ & Prática & $\begin{array}{c}\text { Atividade física de aventura: proposta na } \\
\text { EF escolar para o Ensino Fundamental } \\
\text { de } 3 \text { escolas da rede pública de } \\
\text { Campinas/SP }\end{array}$ \\
\hline Armbrust e Silva ${ }^{20}$ & Teórica & Esportes radicais na EF escolar \\
\hline Alves e Corsino ${ }^{21}$ & Teórica & $\begin{array}{l}\text { Parkour como possibilidade para aulas } \\
\text { de EF escolar }\end{array}$ \\
\hline $\begin{array}{l}\text { Bocchini e } \\
\text { Maldonado }^{22}\end{array}$ & Prática & $\begin{array}{l}\text { Esportes com rodas (bike, patins, skate e } \\
\text { patinetes) em aulas de EF em uma } \\
\text { escola pública de São Paulo/SP }\end{array}$ \\
\hline Tomio et al. ${ }^{23}$ & Teórica & $\begin{array}{l}\text { Esportes radicais como conteúdo } \\
\text { interdisciplinar no contexto escolar }\end{array}$ \\
\hline Tahara e Darido ${ }^{24}$ & Teórica & $\begin{array}{l}\text { O conteúdo das PCA em aulas de EF na } \\
\text { escola }\end{array}$ \\
\hline Inácio et al. ${ }^{25}$ & Teórica & $\begin{array}{c}\text { Relações entre as práticas de aventura } \\
\text { na escola e a BNCC } \\
\text { Avaliação da implementacão de um }\end{array}$ \\
\hline De Freitas et al. ${ }^{26}$ & Prática & $\begin{array}{c}\text { programa de PCA na EF escolar em uma } \\
\text { escola estadual do interior de São } \\
\text { Paulo/SP }\end{array}$ \\
\hline Paixão ${ }^{27}$ & Teórica & $\begin{array}{l}\text { Esporte de aventura como conteúdo } \\
\text { possível nas aulas de Educação Física }\end{array}$ \\
\hline
\end{tabular}

Sabe-se que as PCA apresentam uma grande variedade no que tange aos diferentes tipos de modalidades ou práticas, podendo ocorrer diretamente junto à natureza, como é o caso do surfe, trekking, mergulho, mountain bike, rafting, entre outros, ou mesmo em meio urbano, como é o caso do skate, slackline, escalada em parede artificial, parkour, entre outras práticas.

A própria Base Nacional (BNCC) ${ }^{5}$ retrata a importância de se realizar adaptações no espaço escolar, no propósito de simular as práticas de aventura, ou mesmo de forma real, tornando possível que o aluno experimente a vivência e o aprendizado deste conteúdo.

Diante do amplo repertório de possibilidades de PCA, se abre espaço para compreender que muitas destas manifestações corporais poderiam (e deveriam) ocorrer em âmbito educacional nas aulas de Educação Física, a depender do contexto em que se localiza a escola e das condições físicas, materiais e capacitação docente para efetivar tal inserção.

A tabela 4 ilustra quais os conteúdos das PCA foram abordados nos 126 artigos analisados no "estado da arte" deste estudo, com superioridade das práticas ligadas ao surfe, skate e canoagem.

Se torna importante comentar que alguns artigos analisados não enfocavam conteúdos ou modalidades específicas entre as PCA, apenas retratando-as de forma geral. Por isso, o total de 84 conteúdos, sendo inferior ao total de artigos (126) que mencionavam as PCA.

Além disso, na categoria "Outros conteúdos" houve um número de práticas superior em relação a outros conteúdos expostos individualmente. Tal superioridade deve ao fato de que foram reunidos todos os conteúdos e práticas que tiveram apenas uma única menção entre todos os artigos analisados. Tais conteúdos encontrados nos artigos foram os seguintes: Rafting, Montanhismo, Paraquedismo, Parapente e Arvorismo. 
Tabela 4

Conteúdos enfocados das PCA nos artigos analisados no estado da arte

\begin{tabular}{lc}
\hline Conteúdos enfocados das PCA & $\begin{array}{c}\text { Número de } \\
\text { incidência nos } \\
\text { periódicos }\end{array}$ \\
\hline Surfe & 14 \\
Skate / Canoagem & 11 \\
Escalada & 9 \\
Vela (latismo) & 6 \\
Parkour / Vôo livre & 5 \\
Trilhas interpretativas / Mountain bike & 3 \\
Patins / Slackline / Corrida de Orientação / Corrida de & 2 \\
Aventura / Kitesurf / Rapel & 5 \\
Outros conteúdos & 84 \\
Total & \\
\hline
\end{tabular}

Diante dos resultados referentes aos conteúdos, se percebe a ausência de determinadas práticas que, ao se pensar em uma possível inserção nas aulas escolares, poderia ser bastante exequível a proposta por serem simples e de fáceis adaptações em termos materiais e de espaços físicos.

Nesse sentido, Franco, Cavasini e Darido $^{7}$ apresentam planos de aulas de práticas corporais de aventura, como a Corrida de Orientação, Parkour, Slackline, Arvorismo, Trekking, entre outros, sugerindo propostas de que os professores possam fazer "[...] a contextualização das práticas corporais de aventura de algumas modalidades na estrutura de escolas e espacos comuns, característicos da maioria das regiões do Brasil." (p.114). Os autores ainda enfatizam a importância das PCA em propiciar ao professor a chance de explorar situações relacionadas ao respeito, cooperação entre os pares, vivência de novas experiências corporais e, também, valorização e ética acerca do Meio Ambiente.

\section{Conclusão}

Tomando por base os dados coletados neste "estado da arte" acerca das PCA em onze periódicos nacionais avaliados pelo Quali/Capes na área da Educação Física, considera-se que nesse contexto há ainda uma carência na produção científica relacionada às PCA. Carência ainda maior quando se pensa em uma inserção destas práticas no ambiente escolar, uma vez que tais práticas constituem-se como um conteúdo necessário de ser tratado em aulas, assim como a Base Nacional orienta em suas diretrizes.

Acredita-se que há uma necessidade de realização de mais estudos e pesquisas que incidam foco nas PCA como um conteúdo a ser ministrado nas aulas de Educação Física escolar, haja vista que tais práticas estão cada vez mais inseridas no contexto da sociedade atual.

Afinal, em âmbito ligado ao rendimento, é possível notar nos meios de comunicação de massa um número expressivo de competições e exibições em distintas modalidades que envolvem a aventura, tais como WCT - Surf, Rally Dakar, Campeonatos Mundiais de Rafting, Mountain bike, entre outros.

No que tange ao lazer, se percebe como os indivíduos cada vez mais procuram por tais práticas e vivências em seus momentos de tempo livre, se deslocando aos diversos locais de prática como Brotas/SP, Itacaré/BA, Fernando de Noronha/PE, Socorro/SP, Florianópolis/SC, entre tantos outros destinos nacionais, e muitas vezes pagando por viagens e pacotes a empresas que permitem que a experiência na vivência em determinada modalidade possa acontecer.

Espera-se que as PCA possam se desenvolver academicamente na área da Educação Física, sendo disseminadas com maior frequência em periódicos científicos e na realização de mais estudos e pesquisas que envolvem o tema. Desta forma, se espera que este possa ser um conteúdo cada vez mais tratado e discutido pela área da Educação Física, sendo inserido em contexto escolar, seja no Ensino Infantil, Ensino Fundamental ou Ensino Médio.

\section{Referências}

1. Ontañón $T$, Duprat $R$, Bortoleto $M A$. Educação Física e atividades circenses: "O estado da arte". Movimento 2012; 18(2): 149-168.

2. Rufino LGB, Darido SC. Pesquisa-ação e Educação Física escolar: analisando o estado da arte. Pensar a Prática 2014; 17(1): 242-251.

3. Magrin NP, Simões RMR, Moreira WW. Formação profissional em Educação Física: estado da arte. Kinesis 2014; 32(2): 118-129.

4. Impolcetto FM, Darido SC. O "Estado da Arte" do voleibol e do voleibol na escola. R. bras. Ci. e Mov 2016; 24(4): 175-186.

5. Base Nacional Comum Curricular/BNCC. Ministério da Educação: Brasil. 2017; Disponivel em: <http://basenacionalcomum.mec.gov.br/downloadda-bncc> [2018 mar 03].

6. Pereira DW, Armbrust I. Pedagogia da Aventura: os esportes radicais, de aventura e de ação na escola. Jundiaí: Fontoura, 2010.

7. Franco LCP, Cavasini R, Darido SC. Práticas corporais de aventura. In: González FJ, Darido SC, Oliveira AAB (Org.). Lutas, Capoeira e Práticas corporais de aventura: práticas corporais e a organização do conhecimento. Maringá: Eduem; 2014. p 101-135.

8. Maldonado DT, Silva SAPS. Deportes extremos en la escuela: las tres dimensiones de los contenidos y el desarrollo del pensamiento crítico. Innovación Educativa 2015; 25: 249-267.

9. Tahara AK, Cagliari MS, Darido SC. Celular, Corrida de Orientação, Educação Física escolar: elaboração e avaliação de um material didático. Arq Cien Esp 2017; 5(1): 2-5.

10. Creswell JW. Projeto de Pesquisa: Métodos qualitativo, quantitativo e misto. Porto Alegre: Artmed, 2007.

11. Costa VP, Oliveira FR. A resposta de frequência cardíaca durante as competições de "mountain bike cross-country". Rev. bras. Educ. Fís Esporte 2010; 24(3): 379-387.

12. Tremea VW, Gomes LE, Loss JF. Descrição do comportamento da força do Kite em relação ao seu posicionamento e ao deslocamento da barra de controle. Rev. Bras. Ciênc. Esporte 2011; 33(3): 715-732

13. Manoel EJ, Carvalho YM. Pós-graduação na Educação Física brasileira: a atração (fatal) para a biodinâmica. Educação e Pesquisa 2011; 37(2): 389 406 .

14. Rosa PF, Carvalhinho LAD. A educação ambiental e o desporto na natureza: uma reflexão crítica sobre os novos paradigmas da educação ambiental e o potencial do desporto como metodologia de ensino. Movimento 2012; 18(3): 259-280.

15. Figueiredo JP, Schwartz GM. Atividades de aventura e educação ambiental como foco nos periódicos da área de Educação Física. Motriz 2013; 19(2): 467-479

16. Brandt R, Viana MS, Segato L, Andrade A. Estados de humor de velejadores durante o Pré-Panamericano. Motriz 2010; 16(4): 834-840.

17. Vieira LF, Balbim GM, Pimentel GGA, Hassumi MYSS, Garcia WF. Estado de fluxo em praticantes de escalada e skate downhill. Motriz 2011; 17(4): 591-599.

18. Guimarães SSM, Martins IC, Lucentini L, Carbinatto MV, Moreira WW, Simões R. Educação Física no Ensino Médio e as discussões sobre Meio Ambiente: um encontro necessário. Rev. Bras. Cienc. Esporte 2007; 28(3): 157-172.

19. Franco LCP, Oliveira EC, Oliveira IL, Oliveira MA. Atividades Físicas de Aventura: Proposta de um Conteúdo na Educação Física Escolar no Ensino Fundamental. Arquivos em Movimento 2011; 7(2): 18-35

20. Armbrust I, Silva SAPS. Pluralidade Cultural: os esportes radicais na Educação Física escolar. Movimento 2012; 18(1): 281-300.

21. Alves CSR, Corsino LN. O Parkour como possibilidade para a Educação Física Escolar. Motrivivência 2013; 25(41): 247-257.

22. Bocchini D, Maldonado DT. Andando sobre rodas nas aulas de Educação Física escolar. Motrivivência 2014; 26(43): 277-286.

23. Tomio BW, Silva D, Dalcastagné G, Lamar AR. Os esportes radicais como conteúdo interdisciplinar no contexto escolar. Conexões 2016; 14(1): 104 129.

24. Tahara AK, Darido SC. Práticas corporais de aventura em aulas de Educação Física na escola. Conexões 2016; 14(2): 113-136.

25. Inácio HLD, Cauper DAC, Silva LAP, Morais GG. Práticas corporais de aventura na escola: possibilidades e desafios - reflexões para além da Base Nacional Comum Curricular. Motrivivência 2016; 28(48): 168-187.

26. De Freitas TA, Rufino LGB, Tahara AK, Darido SC. Avaliação da implementação de um programa de práticas corporais de aventura na Educação Física escolar. Arquivos em Movimento 2016; 12(1): 4-16.

27. Paixão JA. Esporte de aventura como conteúdo possível nas aulas de Educação Física escolar. Motrivivência 2017; 29(50): 170-182. 